\title{
Wheel-Rail Contact Relationship Calculation of Worn LMA Tread Feng GAN ${ }^{1, a^{*}}$, Huan-Yun DAI ${ }^{1, b}$, Hao GAO ${ }^{1, \mathrm{c}}$, Mao-Ru CHI ${ }^{1, \mathrm{~d}}$ \\ ${ }^{1}$ Traction Power State Key Laboratory, Southwest Jiaotong University, Chengdu, 610031, China \\ a*ganfengabc@126.com, bdaihuanyun@163.com, 'cgaohao526@163.com, dcmr2000@163.com \\ *Corresponding author
}

Keywords: Rigid Contact, Quasi-elastic Contact, Wheel-rail Contact, LMA Treads, Equivalent Conicity.

\begin{abstract}
Quasi-elastic contact method can be used to study the wheel-rail contact relationship of worn tread. In this paper, wheel-rail contact relationship of standard and worn LMA tread have been calculated under the condition of rigid contact and quasi-elastic contact. By comparing with rigid contact which produces sharp contact points with a large dispersion, quasi-elastic contact results in uniformly smooth contact points without jump, what's more, it's more content with the real wheel-rail contact conditions. And also quasi-elastic contact has a significant effect on the worn tread under different operation mileage. Wheel-rail contact point distribution under different mileage is calculated, and according to tread band, a new measurement of wheelset lateral displacement is developed, which provides a simple way to determine the wheel set lateral displacement only by observing and measuring the tread contact band under different operation mileage. The wheel-rail contact relationship calculation method has been used to the real worn wheel profile test process, where the theory analysis results agreed with the real experiment results well to some extent.
\end{abstract}

\section{Introduction}

With the increasing speed of trains, wheel-rail contact mechanism has become much more complicated. The relationship of wheel-rail interaction is strengthened constantly, which intensifies the wheel-rail wear and makes the tread damage more serious resulting in higher wheel-rail repair costs, and lower quality of interaction between wheel and rail. All that have been mentioned above have a direct influence on the operation stability and safety of high-speed train. Therefore, wheel-rail interaction has drawn more and more attention of railway engineers [1,2].

The basic content of the research on the relationship between wheel and rail is the wheel-rail contact geometry. According to the domestic and foreign researches, various parameters such as wheel and rail shape, rail gauge, rail cant, and the distance between backs of wheel flanges have a direct impact on the wheel-rail contact geometry relationship. Then different wheel-rail contact geometry relationships can produce different wheel-rail forces which will affect the dynamic performance of high-speed trains [3].

The worn wheel-rail contact is one of the most concerned problems in vehicle running process. In order to combine theoretical analysis results with actual worn tread profile test results to optimize the wheel-rail profile matching relationship and adjust vehicle structure parameters constantly to achieve excellent dynamic performance of the vehicle, a large number of researchers are committed to the wheel-rail contact relationship $[4,5]$. The most direct effect of worn wheel and rail is generating out-of-roundness of wheel, wheel diameter deviation and tread shape change which will be accompanied by the change of normal rolling circle radius and equivalent conicity, and these can eventually reflect in the wheel-rail contact geometry relationship. The variation of normal rolling circle radius and equivalent conicity can lead to the deterioration of the dynamic performance of the vehicle [6].

Nonlinear dynamic performance of railway vehicle system is mainly caused by the nonlinear wheel-rail geometry contact relationship. The existing calculation methods of wheel-rail contact geometry relationship rely on the module of commercial software, such as SIMPACK, ADAMS, and VAMPIRE etc. By using space vector mapping algorithm and quasi-elastic method to find wheel-rail contact points, in this paper, software of TPLWRSim is developed to calculate the wheel-rail relationship 
$[7,8]$ and modify the contact point. This software can be used to calculate the wheel-rail contact relationship of any shape of wheel and rail section in real time.

\section{Quasi-elastic Contact of Wheel-rail}

According to the different conditions of wheel tread contacted with rail, the worn shapes of wheel tread are not always the same in the actual vehicle running process. For wheel-rail contact relationship calculation of two wheels in the same wheelset, the rigid contact method which only consider the contact of geometry shape, in one hand, has a fast calculation speed, but is more sensitive to the change of tread profile; on the other hand, doesn't consider the deformation of wheel-rail contact points and has a high degree of contact points position discretion. On the contrary, elastic contact method [9, 10] considering the deformation of wheel-rail contact points has a higher level of accuracy, but it needs to use the finite element analysis and the computing speed is slow and the efficiency is low. In order to reduce the simulation time without losing the accuracy of the calculation, the wheel-rail contact model can be seen as a quasi-elastic contact model $[7,8]$.

The quasi-elastic contact method which considers the deformation of wheel-rail contact points is based on the result of the rigid contact method. In the wheel-rail contact range, firstly, the minimum distances of wheel and rail surface around the wheel-rail rigid contact point are need to be calculated, and then the deformation weights of these minimum distances are calculated by exponentially weighted method, finally, the deformation weights and the transverse distance between approximate contact point and rigid contact point are combined to modify the wheel-rail contact point, therefore the modified contact point is the queasy-elastic contact point.

Assuming that $d(s, q)$ is the minimum distance function of wheel and rail section, where $q$ is a point on the tread, and $s$ is the transverse distance between approximate contact point and rigid contact point, as shown in Figure 1.

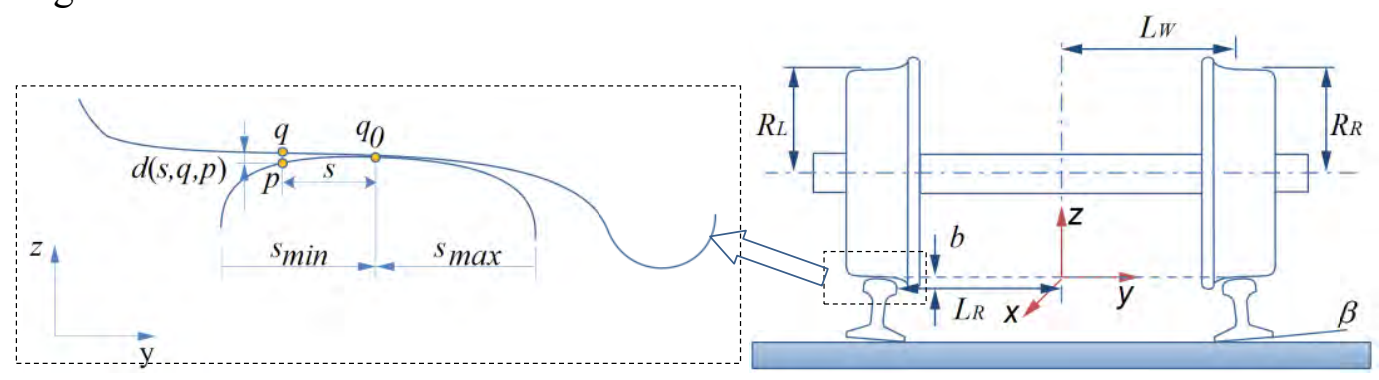

Fig. 1 Wheel-rail Contact Model and Minimum Distance

According to the wheel-rail contact conditions, the minimum distance measured in the contact area is equal to 0 , and the minimum distance measured beyond the contact area is greater than 0 . It can be expressed as:

$$
g(q)=\min _{s}(d(s, q, p))=0
$$

That is:

$$
\left\{\begin{array}{l}
\left.\frac{\partial}{\partial s} d(s, q, p)\right|_{s=0}=0 \\
\left.\frac{\partial}{\partial s} d(s, q, p)\right|_{s \neq 0}>0
\end{array} \quad\left(s \in\left[s_{\text {min }}, s_{\text {max }}\right]\right)\right.
$$

Where $s=q_{x}-q_{0 x}, q_{0}$ is the rigid contact point; $d(s, q, p)=q_{y}-p_{y}$ is the vertical distance between the tread point $q$ and the corresponding point $p$ on the rail surface.

According to the theory of elastic mechanics and logarithmic strain [9], the maximum deformation of wheel and rail surface appears at the contact point, and in the areas outside the contact point, the farther 
the point is away from the contact point, the smaller the deformation is. With the assumption that the relationship of the contact space and deformation of wheel and rail surface is exponential, the weight function can be expressed as:

$$
w(s, q, p)=\exp \left(\frac{-d(s, q, p)}{\varepsilon}\right)
$$

Where, $\varepsilon$ is the logarithmic strain.

The calculation of $\varepsilon$ is as follows:

The distance of two particles around the wheel-rail contact point is $\delta_{0}$, while under a certain load the deformation distance become $\delta_{n}$, and then the relative linear strain is:

$$
\varepsilon_{L}=\frac{d \delta}{\delta_{0}}=\frac{\delta_{n}-\delta_{0}}{\delta_{0}}
$$

According to the principle of material molding, when $d \delta$ is very small, the logarithmic strain is $\varepsilon=\varepsilon_{L}$

When $d \delta$ is large, the logarithmic strain is the integration of linear strain with relative strain process, that is:

$$
\varepsilon=\int_{\delta_{0}}^{\delta_{n}} \frac{d \delta}{\delta}=\ln \frac{\delta_{n}}{\delta_{0}}
$$

For wheel-rail contact, the value of $\varepsilon$ is in the range of $10^{-5} \sim 5 \times 10^{-5}$.

Due to $d(s, q, p) \geq 0$, the value of $w(s, q, p)$ is in the range of $0 \sim 1$.

Combining the deformation weights $w(s, q, p)$ with the transverse distance $s$ between approximate contact point and rigid contact point, the wheel-rail contact point $q_{0}$ can be modified, and the modified formula is defined as follows:

$$
\bar{s}=\frac{\int_{s_{\min }}^{s_{\max }} s \cdot w(s, q, p) d s}{\int_{s_{\min }}^{s_{\max }} w(s, q, p) d s}
$$

After modification, the contact point $q(\bar{s})$ is therefore the queasy-elastic contact point.

\section{Equivalent Conicity Calculation of Harmonic Linearization Method}

Because of wheel and rail wear, track deviation and other factors, the wheel-rail contact geometry has widely dispersed. Then it is necessary to select a simple and reasonable parameter to evaluate the wheel-rail contact geometry relationship. Equivalent conicity as a wheel-rail contact linearization index is typically used to characterize the wheel-rail contact geometry in railway applications [6]. EN14363 [11] and UIC518 [12] standards have been specified the use of this parameter to evaluate the wheel-rail contact geometry. International Union of Railways standard UIC519 [13] has defined nominal equivalent conicity as the value of equivalent conicity for a wheelset's amplitude of $3 \mathrm{~mm}$.

There are many methods to calculate the equivalent conicity. For example, UIC519 standard calculates equivalent conicity by using the hypothesis of wheelset stochastic movement, while SIMPACK software uses the hypothesis of the periodic sinusoidal wheelset movement which is called harmonic linearization method $[13,14]$.

When use harmonic linearization method to calculate the equivalent conicity, a function $\mathrm{y}_{D F}$ described about the wheel diameter difference $\Delta r$ is necessary [15].

In order to linearize the -describing function", it needs to calculate the quadratic error between the nonlinear function $\mathrm{y}_{D F}=f(x)$ and the quasi-linear approach $\mathrm{y}_{D F}=k x$ minimum according to the 
principle of least square method.

$$
\Delta^{2}=(f(x)-k x)^{2}
$$

That is:

$$
\frac{\partial}{\partial k}\left(\Delta^{2}\right)=0
$$

After a differentiation, the coefficient $k$ can be expressed as:

$$
k=\frac{x \cdot f(x)}{x^{2}}
$$

Assuming the solution is a harmonic expression with amplitude A, that is:

$$
x(w t)=A \cdot \sin w t
$$

Then the linear factor $k(A)$ of the -describing function" which is related with the harmonic linearization amplitude A can be obtained.

$$
k(A)=\frac{1}{\pi \cdot A} \int_{o}^{2 \pi} f(A \cdot \sin \varphi) \cdot \sin \varphi d \varphi
$$

Assuming the -describing function" of the wheel rolling radius difference is $\Delta r=f(y)$, and then the expression of equivalent conicity with the linear amplitude A can be expressed as follows:

$$
\lambda(A)=\frac{1}{2 \pi A} \int_{o}^{2 \pi} \Delta r(A \cdot \sin \varphi) \cdot \sin \varphi d \varphi
$$

Form the equation above, equivalent conicity can be obtained by the numerical integration of the nonlinear equation about the wheel diameter difference $\Delta r$.

\section{Wheel-rail Contact Relationship of LMA Tread}

LMA tread is China Railway Standard tread, and the tread profile is shown in Figure 2. Software of TPLWRSim which uses space vector mapping algorithm to find wheel-rail contact points and uses quasi-elastic method to modify the contact point is developed to calculate the wheel-rail relationship, and the software interface is shown in Figure 3. Calculation parameters of wheel-rail contact relationship are shown in Table 1.

Tab. 1 Calculation Parameters of Wheel-rail Contact

\begin{tabular}{ll}
\hline Parameter & Value \\
\hline Tread type & LMA \\
Rail type & CHN60 \\
Nominal rolling radius $(\mathrm{mm})$ & 430 \\
Back-to-back distance of the wheelset $(\mathrm{mm})$ & 1353 \\
Track gauge $(\mathrm{mm})$ & 1435 \\
Rail cant & $1 / 40$ \\
Yaw angle $\left({ }^{\circ}\right)$ & 0 \\
Lateral displacement range of wheelset $(\mathrm{mm})$ & $0 \sim 12$ \\
Step of lateral displacement $(\mathrm{mm})$ & 0.1 \\
Flange thickness $(\mathrm{mm})$ & 32 \\
Flange height $(\mathrm{mm})$ & 28 \\
\hline
\end{tabular}




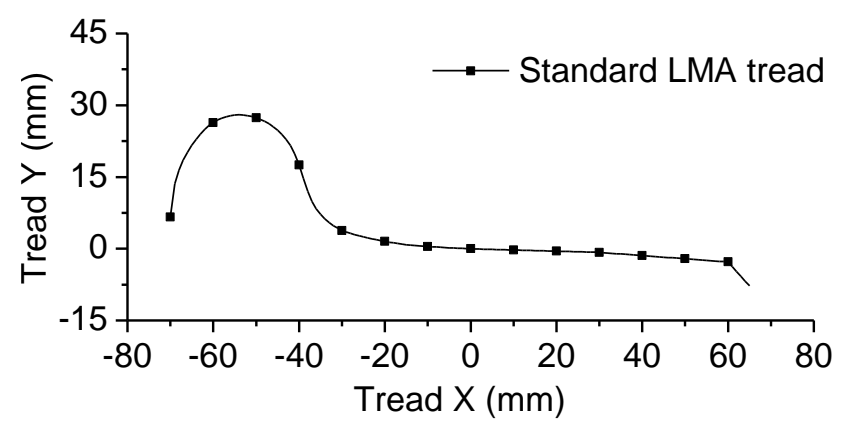

Fig. 2 Standard LMA Tread Graph
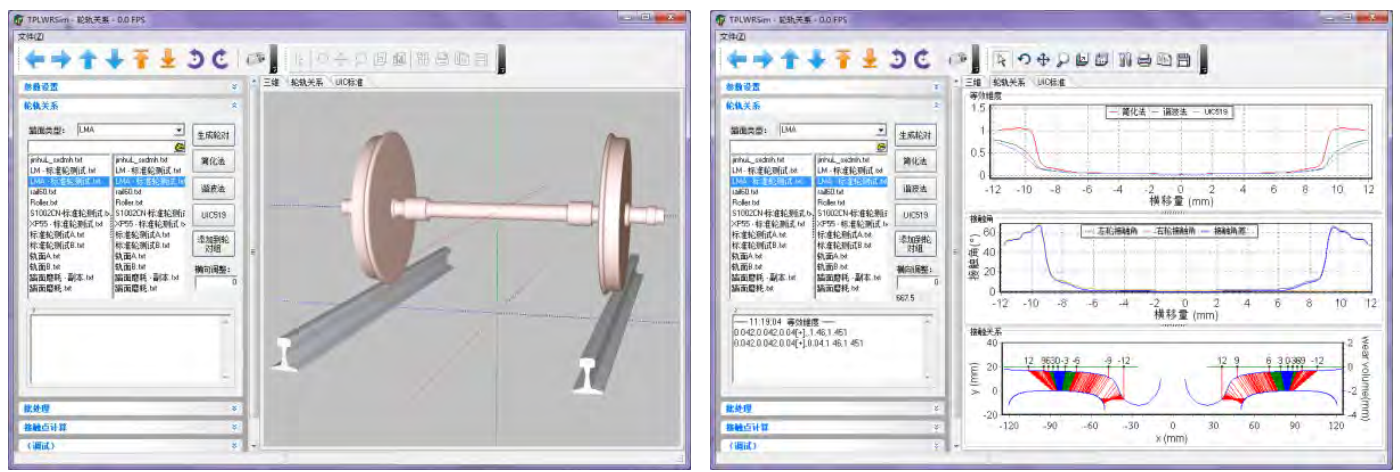

Fig. 3 TPLWRSim Software Interface

In the actual operation process of EMU, the wear situation of left and right wheel in the same wheelset is inconsistent, and especially for a bogie or a vehicle, the wear situation of each wheel is also not the same. In this paper, 4 groups of test data have been extracted from the large amounts of measured wheel tread data in the operation period of a certain EMU, and these test data are used to study the trends of the actual tread shape, wheel diameter difference, equivalent conicity, wheel-rail contact relationship and the contact point distribution on the tread.

\section{Comparison of the Rigid Contact with Quasi-elastic Contact of Wheel-rail}

According to Table 1, by using the rigid and quasi-elastic contact algorithm, respectively, the wheel diameter difference, equivalent conicity and wheel-rail contact relationship of standard LMA tread and worn LMA tread have been calculated and the results are shown in Figure 4.

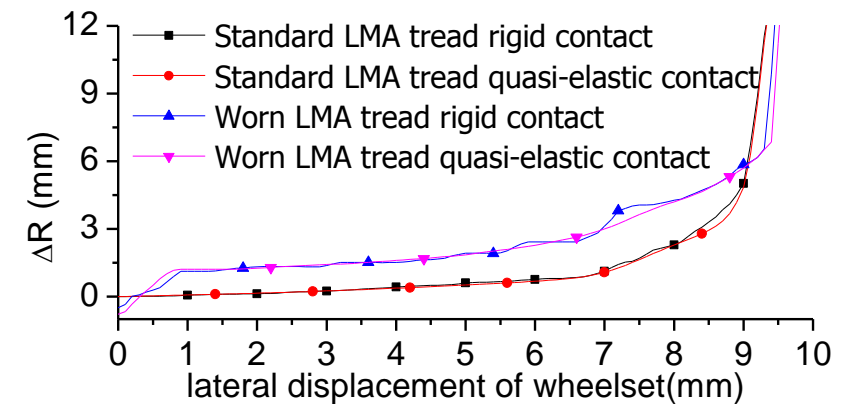

(A) Wheel diameter difference diagram

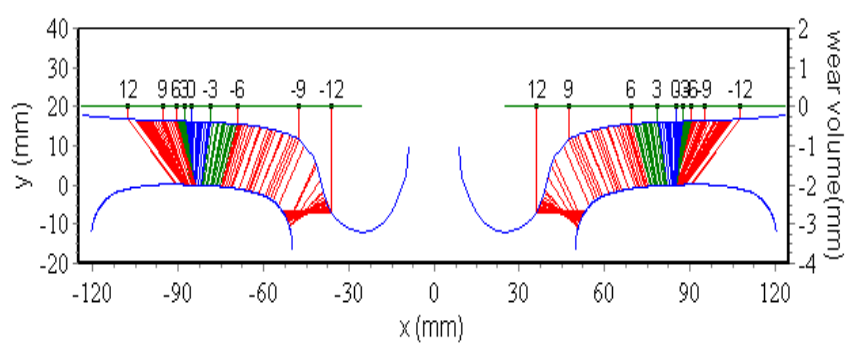

(C) rigid contact Standard LMA tread

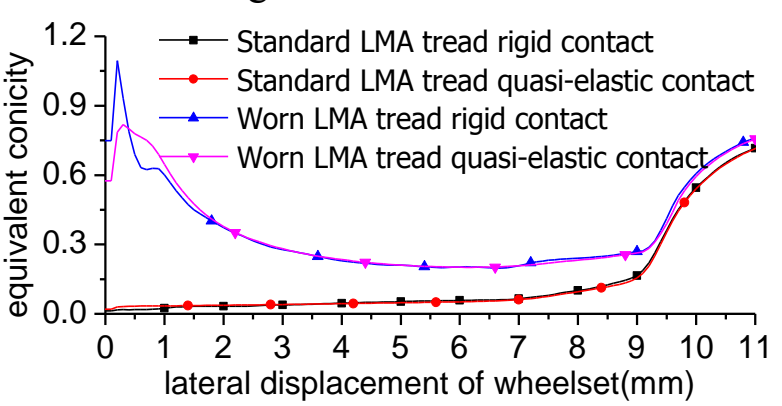

(B) Equivalent conicity diagram

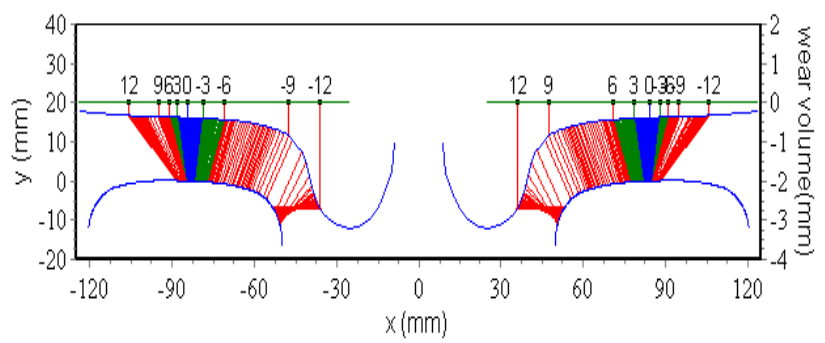

(D) quasi-elastic contact of Standard LMA tread 


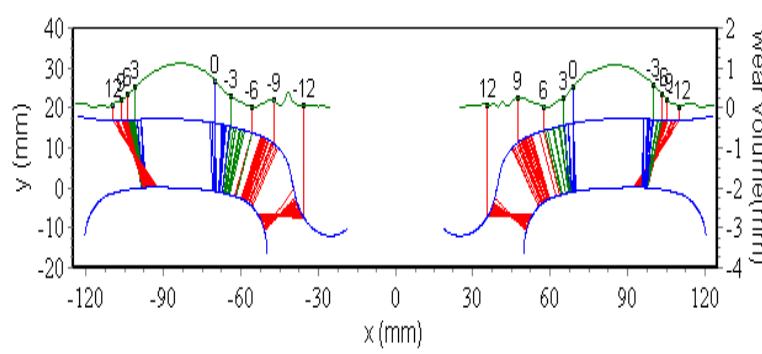

(E) rigid contact of Worn LMA tread

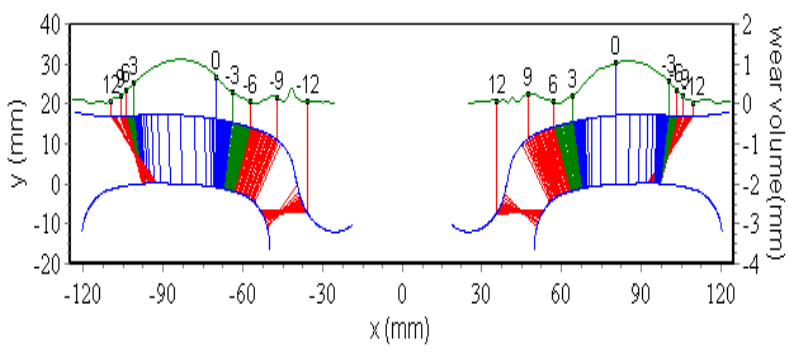

(F) quasi-elastic contact of worn LMA tread

Fig. 4 Wheel-rail Contact Relationships of LMA Tread

The blue lines indicate the positions of wheel-rail contact points when wheelset moves laterally from -3 $\mathrm{mm}$ to $3 \mathrm{~mm}$ respect to its central line; The green lines indicate the positions of wheel-rail contact points when wheelset moves laterally from $-6 \mathrm{~mm}$ to $-3 \mathrm{~mm}$ and from $3 \mathrm{~mm}$ to $6 \mathrm{~mm}$ respect to its central line; The red lines indicate the positions of wheel-rail contact points when wheelset moves laterally from -12 $\mathrm{mm}$ to $-6 \mathrm{~mm}$ and from $6 \mathrm{~mm}$ to $12 \mathrm{~mm}$ respect to its central line. And the wear curve (green line) of the worn wheel tread in figure 4 is relative to the shape of standard wheel tread.

As the results of rigid and elastic contact are shown in Figure 4, the following conclusions can be drawn:

1. Rigid contact produces sharp contact point with a large dispersion.

2. Quasi-elastic contact produces uniformly smooth contact points without jump. What's more, it's more content with the real wheel-rail contact conditions.

3. The modified quasi-elastic contact has a significant effect on worn tread.

\section{Wheel-rail Contact Relationship of Worn LMA Tread}

By using WA+ type railway wheel profile measuring instrument, the wheel tread profiles under 4 operation mileages about 40000, 90000,150000 and $200000 \mathrm{~km}$ have been measured in the operation period of a certain EMU, respectively. The measurement results under different operation mileage are shown in Figure 5. And the left and right tread wear volumes calculated by radial wear algorithm under different mileage are shown in Figure 6.

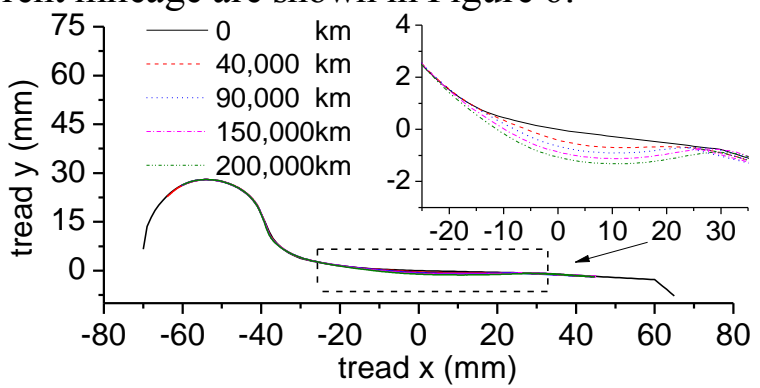

(A) Left wheel tread profile

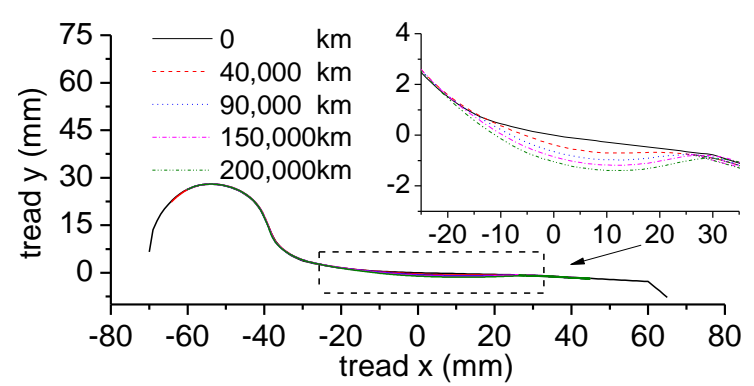

(B) Right wheel tread profile

Fig. 5 Measured LMA Tread Profile under Different Operation Mileage

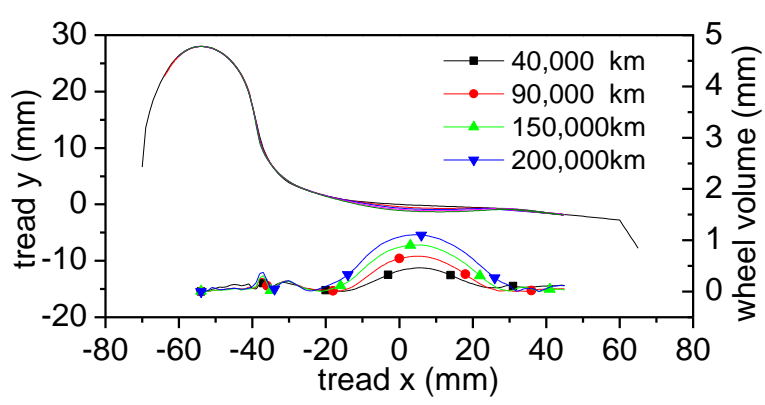

(A) Left Wheel wear curve

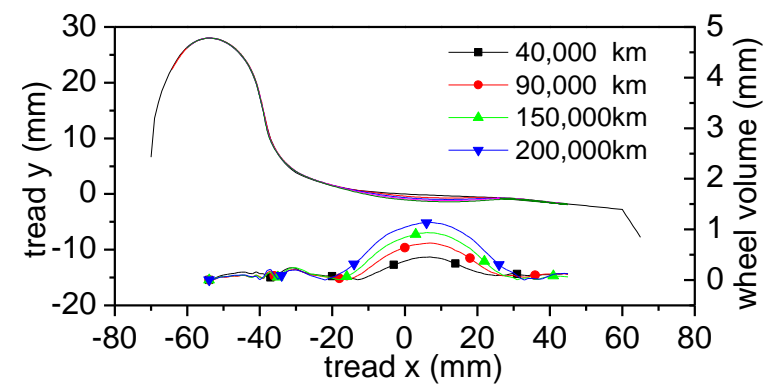

(B) Right Wheel wear curve

Fig. 6 Wear Curve of LMA Tread under Different Operation Mileage 
Then the following conclusions can be drawn from the results of Figure 5 and 6 :

1. The worn type of LMA tread is concave wear, and wear areas are mainly concentrated on the nominal rolling circle;

2. The range of tread wear is from -20 to $30 \mathrm{~mm}$;

3. The amount of tread depression increases with operation mileage. New wheel tread wears faster than the worn wheel tread, and the wear volume of the worn wheel tread almost increases linearly with operation mileage.

The calculation results of wheel diameter difference, contact angle and equivalent conicity under different operation mileage are shown in Figure 7.

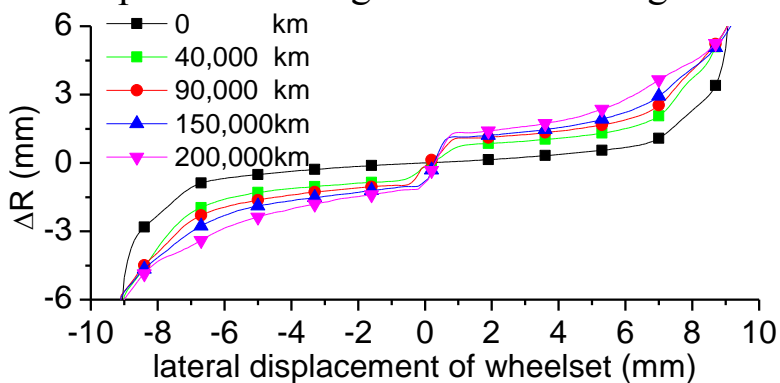

(A) Wheel diameter difference curve

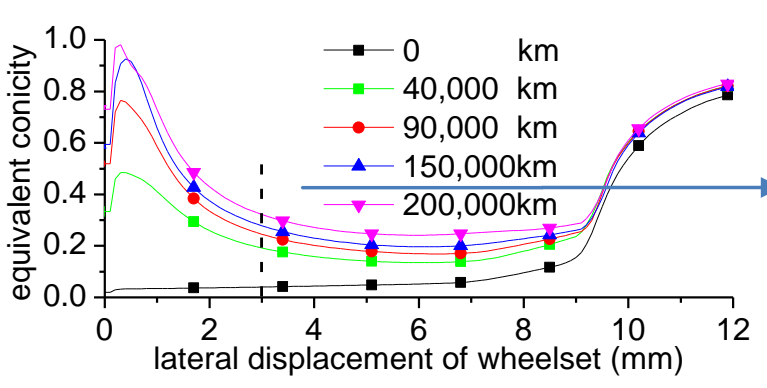

(C) Equivalent conicity curve

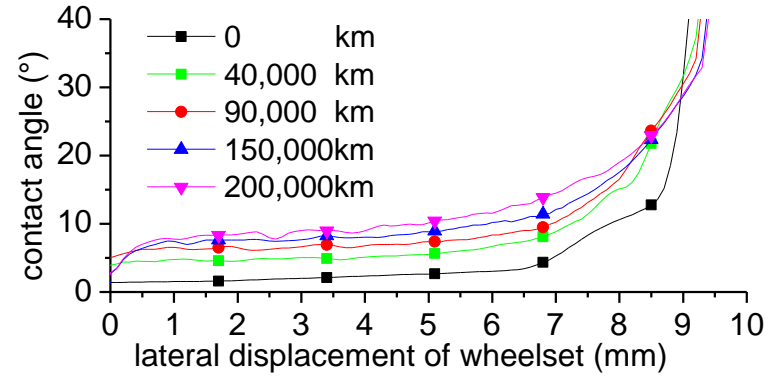

(B) Contact angle curve

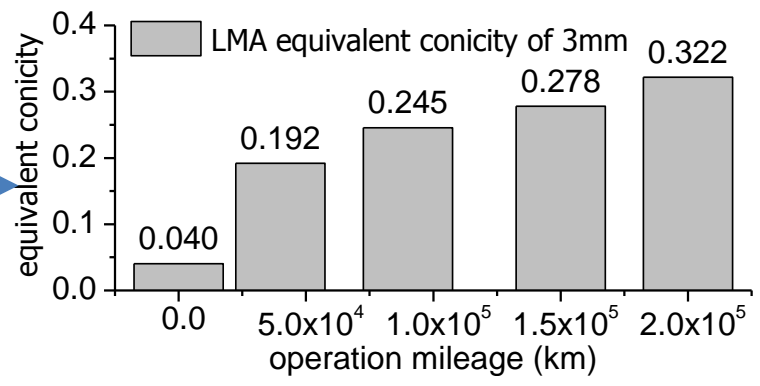

(D) Equivalent conicity of $3 \mathrm{~mm}$ curve

Fig. 7 Wheel-rail Contact Relationship of Worn LMA Tread under Different Operation Mileage

And the wheel-rail contact geometry relationship of worn LMA tread under difference operation mileage is shown in Figure 8.

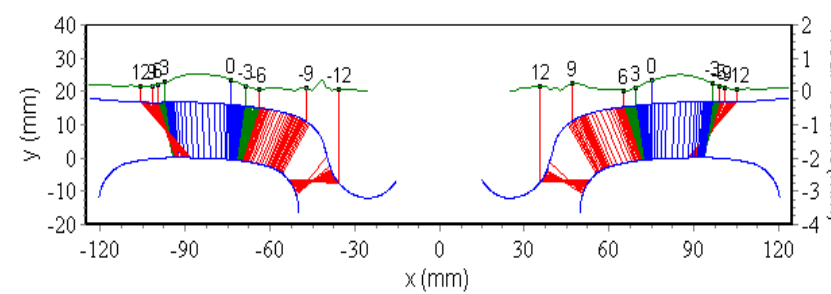

(A) $40,000 \mathrm{~km}$ operation mileage

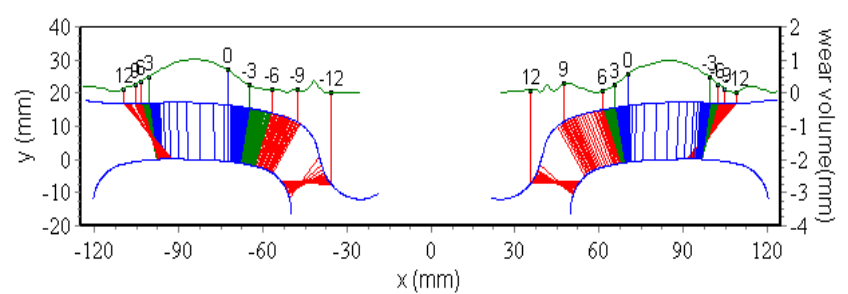

(C) $150,000 \mathrm{~km}$ operation mileage

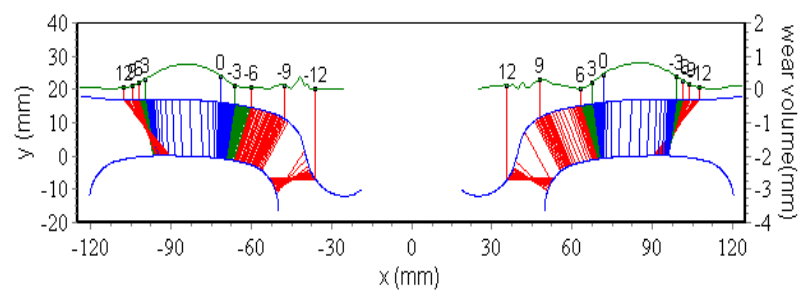

(B) $90,000 \mathrm{~km}$ operation mileage

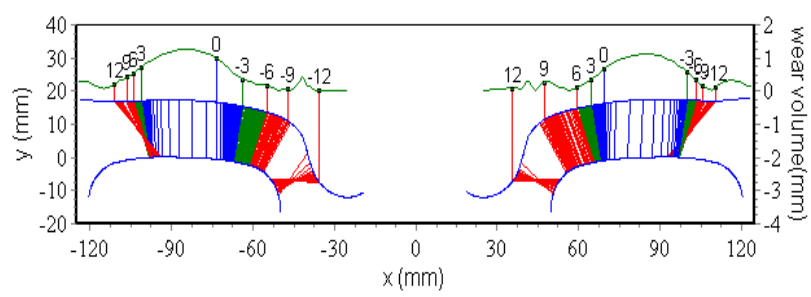

(D) $200,000 \mathrm{~km}$ operation mileage

Fig. 8 Wheel-rail Contact Geometry Relationship of Worn LMA Tread under Different Operation Mileage

The wheel-rail contact point distribution of LMA tread under different operation mileage is shown in Figure 9. 

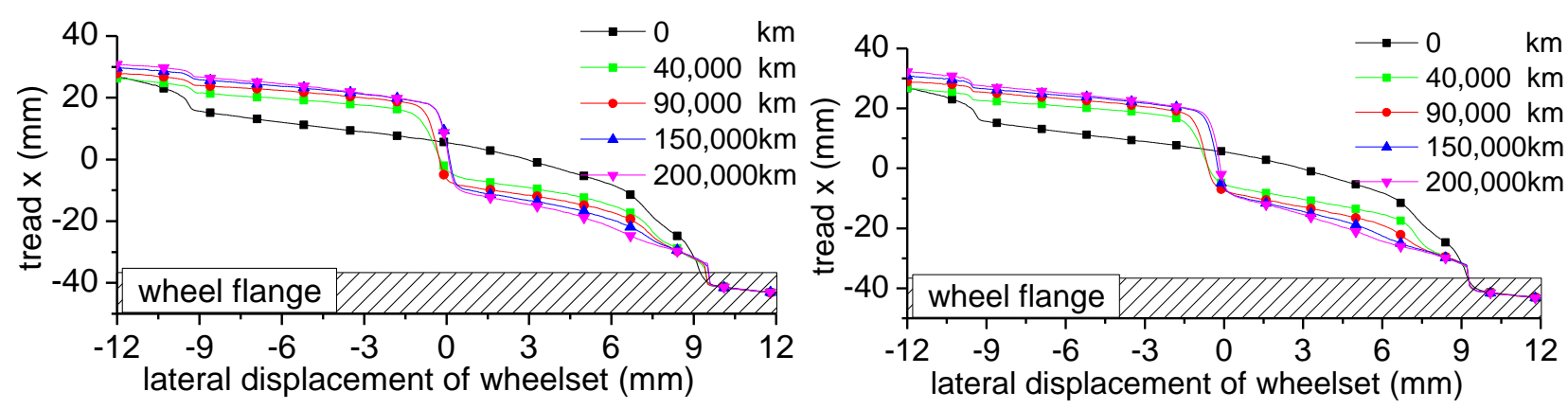

(A) Left wheel contact point variation curve

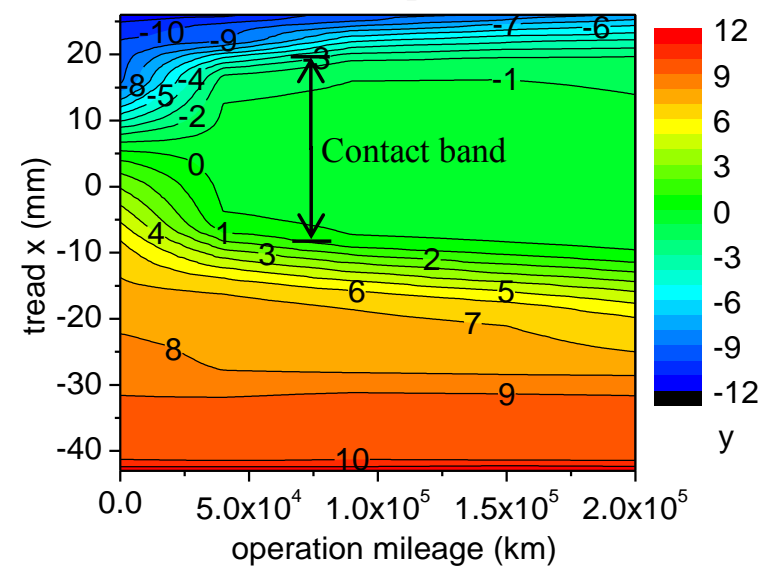

(B) Right wheel contact point variation curve

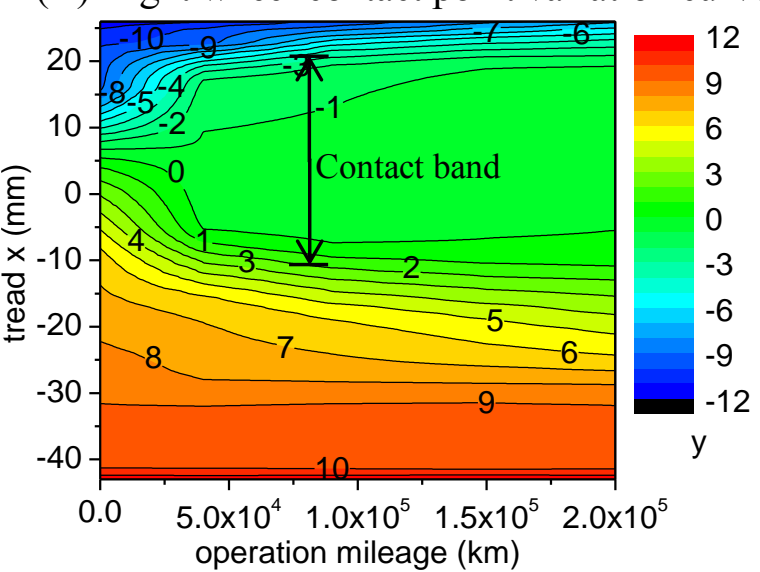

(C) Left wheel contact point distribution

(D) Right wheel contact point distribution

Fig. 9 Wheel-rail Contact Point Distribution of LMA Tread under Different Operation Mileage

As the results of rigid and elastic contact are shown in Figure 7 9, the following conclusions can be drawn:

1. Wheel diameter difference, contact angle and equivalent conicity increase with operation mileage. And equivalent conicity of the new wheel tread grows faster than the worn wheel tread, and the equivalent conicity of the worn wheel tread almost increases linearly with operation mileage which can be seen from Figure 7.

2. From the result of wheel-rail contact relationship of worn LMA tread under different operation mileage, as shown in Figure 8, the distribution of contact point modified by quasi-elastic contact method is very uniform which also proves the accuracy and validity of quasi-elastic method. With the operation mileage increasing, when the lateral displacement of wheelset is from $-3 \mathrm{~mm}$ to $3 \mathrm{~mm}$, the moving speed of the contact point is faster and faster in the constant wheel-rail contact area.

3. Figure 9 shows the distribution of wheel-rail contact point of LMA tread under different operation mileage. It can be seen that the contact band width of wheel and rail contact area becomes wider and wider with the increasing of the operation mileage. In this case, according to tread contact band attained by searching the band width and position in the Figure 9 (C) and (D), a new measurement method of wheel set lateral displacement, which provides a sample way to determine the wheel set lateral displacement only by observing and measuring the tread band under different operation mileage, is developed.

\section{Summary}

Quasi-elastic contact method, which produces uniformly smooth contact points without jump and is more content with the real wheel-rail contact conditions, can be used to study the wheel-rail contact relationship of worn tread. What's more, quasi-elastic contact can also have a significant effect on the worn tread under different operation mileage.

Wheel-rail contact point distribution under different mileage is calculated, and a new measurement method of wheel set lateral displacement, which provides a sample way to determine the wheel set lateral displacement only by observing and measuring the tread band under different operation mileage, is 
developed. The wheel-rail contact relationship calculation method in this paper has been used to the real worn wheel profile test process where the theory analysis results agreed with the real experiment results well to some extent.

\section{Acknowledgement}

The authors would like to acknowledge the supports from the National Natural Science Foundation of China (Grant 51005189), the National Basic Research Program of China (Program 973) (Grant 2011CB711106)

\section{References}

[1]JIN Xue-song, SHEN Zhi-yun. Rolling contact fatigue of wheel/rail and its advanced research progress. Journal of the China Railway Society, 2001, 23(2): 92-108.

[2]JIN XueSong, ZHANG XueShan, ZHANG Jian, etal. Mechanics in performance of wheel-rail. Journal of Mechanical Strength, 2005, 27(4):408-418.

[3]LUO Ren, ZENG Jing, WU Ping-bo, etal. Influence of wheel/rail parameters on wheel profile wear of high-speed train. Journal of Traffic and Transportation Engineering, 2009, 9(6):47-63.

[4]Sergey Zakharov, Irina Goryacheva, Victor Bogdanov, etal. Problems with Wheel and Rail Profiles Selection and Optimization[J].Wear, 2008, 265(9-10):1266-1272.

[5]LI Yan, ZHANG Wei-hua, CHI Mao-ru, etal. Influence of Wheel Tread Profile and Rolling Diameter Difference on Dynamic Performance of Vehicles. JOURNAL OF THE CHINA RAILWAY SOCIETY, 2010, 32(1):104-108.

[6]Oldrich Polach, Characteristic parameters of nonlinear wheel/rail contact geometry. Vehicle System Dynamics, Vol.48, Supplement, 2010, pp.19-36.

[7]Gunter S., Christoph W., Lutz M., Modelling the Contact Between Wheel and Rail Within Multibody System Simulation [J], Vehicle System Dynamics, 2004, 41(5): 349-364.

[8]NetterH, Schupp G, Rulka W, et al. New Aspects of Contact Modelling and Validation within Multibody System Simulation of railway Vehicles [J]. Vehicle System Dynamics Supplement, 1998, (28): $246-269$.

[9]XU ZhiLun. Elastic mechanics, 4thed. Higher Education Press, 2006.7.

[10]Johnson, K. L., Contact Mechanics. Cambridge etc., Cambridge University Press.

[11]EN14363 Railway applications-Testing for the acceptance of running characteristics of railway vehicles-Testing of running behavior and stationary tests. CEN, Brussels, 2005.

[12]UIC Code 518: Testing and approval of railway vehicles from the point of view of their dynamic behavior-Safety-Track fatigue - Ride quality. International Union of Railways, 3rded, Paris, October 2005.

[13]UIC Code 519: Method for determining the equivalent conicity. International Union of Railways, 1sted. Paris, December 2004.

[14]prEn 15302: Railway applications - Method for determining the equivalent conicity. Final Draft CEN, November 2007.

[15]Oldrich Polach, Influence of wheel/Rail contact geometry on the behavior of a railway vehicle at stability limit. ENOC-2005, Eindhoven, Netherlands, 7-12 August 2005. 